\title{
Vibrotactile Target Saliency
}

\author{
Alexander Toet ${ }^{* 1}$, Eric L.Groen ${ }^{1}$, Marjolaine Oosterbeek ${ }^{1}$, Ignace T.C. Hooge ${ }^{2}$ \\ ${ }^{1}$ TNO Human Factors, P.O. Box 23, 3769 ZG Soesterberg, The Netherlands \\ ${ }^{2}$ Helmholtz Institute, Utrecht University, Heidelberglaan 2, 3584 CS Utrecht, The Netherlands
}

\begin{abstract}
We tested the saliency of a single vibrotractile target $(\mathrm{T})$ among 2 to 7 nontargets $(\mathrm{N})$, presented by 8 tactors that were equally distributed over a horizontal band around the torso. Targets and nontargets had different pulse duration, but the same activation period and no onset asynchrony. T-N similarity was varied by changing the difference between $\mathrm{T}$ and $\mathrm{N}$ pulse duration. For target present trials the response times increased with the number of stimulus items for all conditions tested, suggesting a serial discrimination process. For target absent trials the response times were independent of the number of stimulus items, suggesting a parallel discrimination process. We found no effect of T-N similarity and no search asymmetry. The present results suggest that tactile target search is not comparable to visual search.
\end{abstract}

Keywords: parallel search, serial search, saliency, tactile display, target detection

\section{INTRODUCTION}

In order to design effective tactile information displays one needs to know how well a tactile target can be detected amongst other simultaneously presented tactile stimuli ${ }^{1}$. For the visual capability, much is known about the detection of target signals among distracting nontarget signals. However, for the tactile modality this knowledge is currently lacking.

Tactile devices and tactile displays have become increasingly popular in recent years, since they can provide relief from auditory, visual, and cognitive sensory overload ${ }^{2-7}$. Body-worn spatially-referenced tactile displays ("body displays") can present directional information about orientation, localized targets, threats, friends, objects etc. in a way that is more intuitive than presentation of the same information through the visual and auditory pathways, and may therefore require less cognitive capacity. Tactile stimulus characteristics that can be used to code information are movement, movement direction, movement orientation, movement speed, movement type, duty cycle, rhythm, rhythm frequency, rhythm phase, location, and different combinations of these characteristics. Tactile displays seem to be especially suited for navigation and orientation tasks ${ }^{2-5}$. Moreover, tactile signals are highly suitable as warning signals, since they have the potential to draw direct attention ${ }^{2,8}$. Promising aeronautical applications include directional threat warnings and support during low level flight and hover tasks. Tactile displays consisting of a matrix of actuators can present multiple tactile stimuli simultaneously. When presenting multiple information streams in parallel, the characteristics of the elementary stimuli should be such that the independent tactile information streams are optimally detectable and distinguishable, and that they minimally interfere with each other. From the human visual modality we know that simultaneously presented stimuli can significantly influence each other's detectability (masking, lateral inhibition, contour interaction) or perceived position or orientation (crowding) ${ }^{9,10}$. However, it is currently not known if similar effects exist in the tactile sensing modality. Thus, we do not know if a specific tactile target (e.g. a warning signal) can still draw attention (i.e. is remains salient) when presented in conjunction with other tactile signals.

In the visual domain, multiple simultaneously presented stimuli are not processed independently, but rather interact in a mutually suppressive way, suggesting that they compete for neural representation in the visual cortex ${ }^{11}$. This competition can be influenced by both top-down (attention) and bottom-up (stimulus driven) mechanisms. For example, if one directs attention to a particular location in a cluttered scene, processing of information at the attended location will be facilitated and processing of unwanted information from nearby irrelevant objects (distracters) will be suppressed, suggesting that competition is biased in favour of the attended stimulus. On the other hand, if a salient stimulus is present in a cluttered scene, it will be effortlessly and quickly detected regardless of the number of distracters, suggesting that competition is

\footnotetext{
* lex.toet@tno.nl; phone +31-346-356237; fax +31-346-353977; http://lextoet.googlepages.com
} 
biased in favour of the salient stimulus. In the visual domain, suppressive cortical interactions among multiple stimuli are eliminated when they are presented in the context of pop-out displays, in which a single item differs from the others, but not in heterogeneous displays, in which all items differ from each other ${ }^{11,12}$.

In vision we distinguish parallel versus serial search. Parallel search yields detection times that are independent of the number of nontargets, whereas detection times for serial search increase with the number of nontargets (although this distinction has recently been challenged ${ }^{13}$ ). Parallel search is thought to reflect the preattentive processing of individual features, whereas serial search is thought to result from the attentive processing of conjunctions of features ${ }^{14}$ Target saliency plays an important role here: a salient target will be detected fast, independent of the number of nontargets, because it provokes feature search, instead of conjunction search ${ }^{15}$. However, even feature search can become serial when the similarity of targets to nontargets increases (i.e. when the target saliency decreases) and when the similarity between nontargets decreases ${ }^{16,17}$. Hence, parallel and serial processes are not necessarily isolated, but fall along a continuum ${ }^{18}$ For high N-N similarity, nontargets can be suppressed as a group, against which the target is detected easily. For low N-N similarity, it is harder to suppress the nontargets as a group and they require individual evaluation, i.e. serial processing ${ }^{19}$.

Response times for visual signal detection may differ dramatically when the roles of target and of distractors are interchanged. This phenomenon is known as search asymmetry ${ }^{20}$. The relative salience of features in the two signals plays an important role in search asymmetry: the distinctive features of the more salient figure are given greater weight in determining dissimilarity. Also, if a target possesses a certain feature that the nontargets do not have, search asymmetry can be observed when the roles of the target and the nontargets are exchanged ${ }^{19}$.

One of the key concepts in vibrotactile target detection is the masking phenomenon ${ }^{21}$. Masking refers to the fact that the percept of a stimulus (target) may change when another stimulus (masker) is presented close in time or space ${ }^{2,22}$. In this study however, we do not intend to mask stimuli, but we want to test if tactile target distinctness depends on the number of nontargets. Because little is known about this aspect of tactile signal processing, this study will be based on what is known about signal detection in the human visual system. Attempts have already been made to analyze tactile perception in terms of the known mechanisms of the visual system ${ }^{23,24}$. Although the visual and tactile senses indeed appear to share some common processing strategies ${ }^{25,26}$, it evident that the visual system works inherently differently from the tactile modality ${ }^{24,27}$.

In this study we investigated if the signal detection properties of the haptic sensing modality are similar to those of the human visual system. We used small vibrating elements (tactors) to deliver localised tactile signals to the skin. Targets and nontargets had different pulse duration, but the same activation period and no onset asynchrony. T-N similarity was varied by changing the difference between $\mathrm{T}$ and $\mathrm{N}$ pulse duration. We varied the number of nontargets to test whether tactile stimulus discrimination shows parallel and serial processing modes, and we interchanged the roles of targets and nontargets to investigate if there is a search asymmetry.

The rest of this paper is as follows. First we present the stimuli and procedures used in this study. Then we present the results of the experiments. Finally we discuss these results and we will give some suggestions for further research.

\section{METHOD}

Six male participants in the age ranging from 22 to 42 volunteered for this study. The experimental protocol was approved by TNO Human Factors internal review board on experiments with human participants. The participants gave their informed consent prior to testing.

The vibrating elements or tactors we used were custom built, and are based on small DC pager motors that are housed in a PVC contactor, with a contact area of 1.5 by $2.0 \mathrm{~cm}$. They vibrate at a frequency of $150 \mathrm{~Hz}$, stimulating mainly the Pacinian Corpuscles in the skin. An electronics unit connects the tactors with the parallel port of a standard PC. Special purpose software allows for individual on/off control of each tactor at up to $250 \mathrm{~Hz}$ update rate, but in practice the DC motors take a finite time to start and stop and the effective update rate is down to roughly $50 \mathrm{~Hz}$.

Eight tactors were attached with sticking plaster directly to the skin of the participant's abdomen, starting $1 \mathrm{~cm}$ below the navel to all around the abdomen, at equal spatial intervals. When activated, they administered a localized vibration, resulting in a sensation on the skin that was similar to a vibrating mobile phone. Participants' circumferences were never 
smaller than $80 \mathrm{~cm}$, so the distance between tactors always was at least $10 \mathrm{~cm}$, i.e. larger than a single receptive field (about $4 \mathrm{~cm}^{2}$ ). To mask auditory cues of the tactor vibration the participants wore headphones which produced pink noise of $65 \mathrm{dBA}$.

The experimental design was similar to that commonly applied in studies of target saliency in the visual domain (e.g. studies on parallel versus serial search, lateral interaction, masking, crowding or pop-out). This means that multiple tactile stimuli were presented simultaneously, and we measured the accuracy and speed with which the subject could detect the presence or absence of a designated target stimulus, as a function of the number of nontarget stimuli. Tactile targets and nontargets differed in their on/off rhythm only. Targets were always presented simultaneously with nontargets. In a target-present trial, one of the tactors (the target) vibrated in a rhythm that differed from that of the nontargets. Onset times of targets and nontargets were the same, so that stimulation by both was simultaneous without phase differences. To rule out spatial interaction effects, active tactors always formed a consecutive series (Fig. 1), resulting in a fixed inter-tactor spacing. Both targets and nontargets were activated during a period of $2000 \mathrm{~ms}$ (the maximum allowed response time). Within four different conditions the set size (i.e. the number of active tactors) was varied randomly: per trial, either 3, 4, 6 or 8 tactors vibrated, at random places.

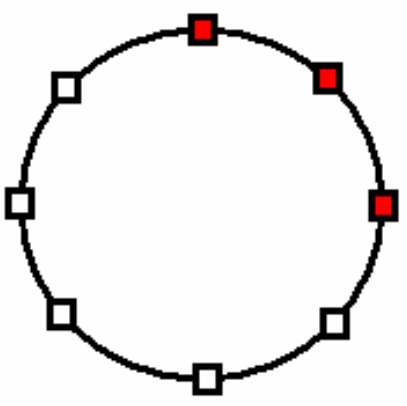

(a)

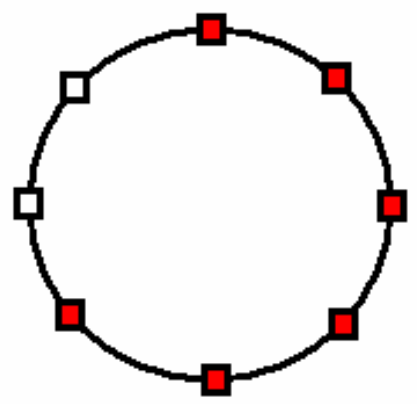

(c)

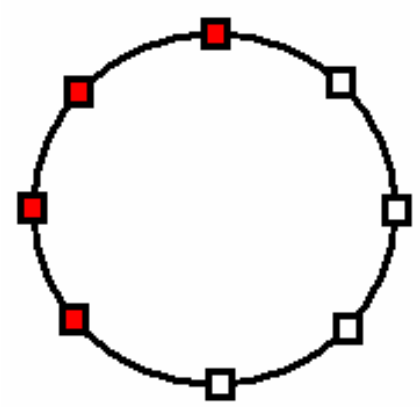

(b)

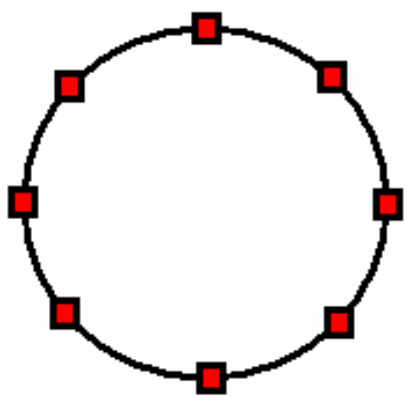

(d)

Fig. 1. Schematical representation of the positions of all 8 tactors (squares) around the participant's abdomen. Filled squares represent activated tactors. Stimulus set size was either 3 (a), 4 b), 6 (c) or 8 (d). 


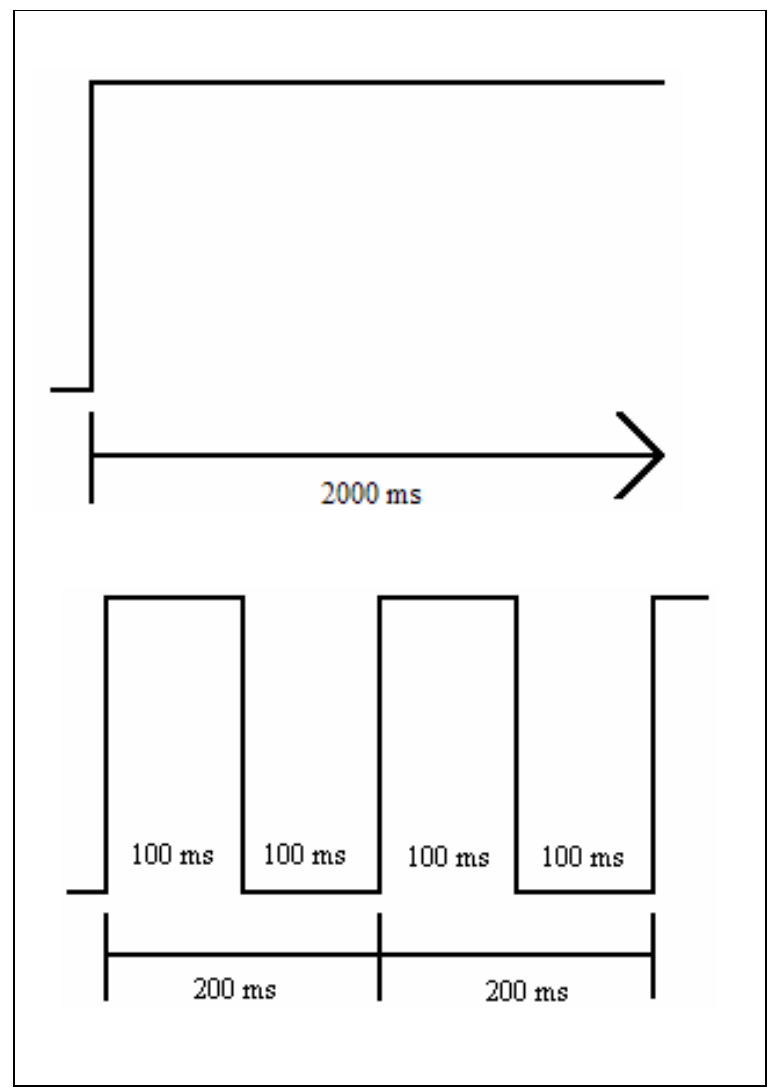

(a)

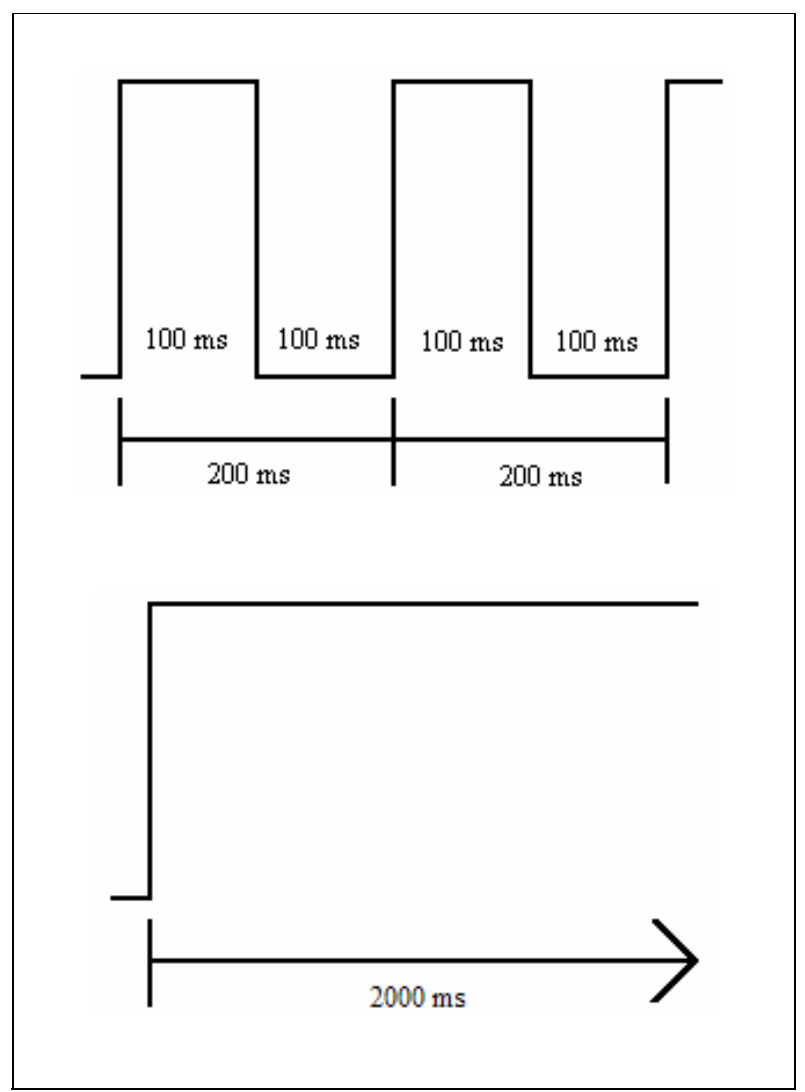

(b)

Fig. 2. Temporal activation patterns of targets and non-targtes in the low T-N similarity (left column) and the high T-N similarity conditions (right column).

There were four experimental conditions, allowing for a 2 x 2 within subject design: two levels of T-N similarity (low and high similarity) and two levels of T-N roles (interchanged or not). The presentation order of the four conditions was counterbalanced over participants using a Latin square design ${ }^{28}$. The tested conditions and their corresponding tactile stimuli were as follows:

1. Low T-N similarity (Fig. 2a).

○ The nontargets were a continuous tactile stimulation.

- The target had an on-period or burst duration (BD) of $100 \mathrm{~ms}$, followed by an off-period of $100 \mathrm{~ms}$, resulting in a repeating sequence of pulses with a 'cycle time' of $200 \mathrm{~ms}$.

2. Low T-N similarity - interchanged (Fig. 2b).

In this condition, the roles of the target and nontargets in condition 1 were interchanged:

- The nontargets had a BD of $100 \mathrm{~ms}$ with a cycle time of $200 \mathrm{~ms}$.

○ The target was a continuous tactile stimulation with a duration of $2000 \mathrm{~ms}$. 
In the following two conditions, the target - nontarget similarity was higher to test if the target would be harder to detect.

3. High T-N similarity (Fig. 3a).

- The nontargets had a BD of $200 \mathrm{~ms}$ with a cycle time of $400 \mathrm{~ms}$, i.e. a repeating sequence of pulses with on- and off- periods of both $200 \mathrm{~ms}$.

- The target had a BD of $100 \mathrm{~ms}$ with a cycle time of $200 \mathrm{~ms}$.

4. High T-N similarity - interchanged (Fig. 3b).

In this condition, the roles of the target and nontargets in condition 3 were interchanged:

- The nontargets had a BD of $100 \mathrm{~ms}$ with a cycle time of $200 \mathrm{~ms}$.

○ The target had a BD of $200 \mathrm{~ms}$ with a cycle time of $400 \mathrm{~ms}$.

Participants first performed a practice series consisting of 64 trials, to familiarize themselves with the task and the stimuli, and to reduce possible performance improvement due to training during the actual experiment. This practice series was divided into 4 consecutive blocks of 16 trials each. Each block consisted of stimuli belonging to one of the 4 different conditions. Before running a practice block for the upcoming condition, the experimenter explained the difference between the target-absent and target-present trials, and let the participants experience a few example trials. After that, the practice block started.

After completing the practice series, the participants were presented with three experimental series of 64 trials each: 4 blocks of 16 trials. Again, each block consisted of stimuli belonging to a single one of the 4 different conditions. Trials were randomized within blocks, and the 4 different conditions were randomized within each series. Target-absent and target-present trials both had a prevalence of $50 \%$. The participants were informed that targets could either be absent or present, but they were naive about the actual target/nontarget ratio.

During the experiment, the participants were seated on a chair. Their right and left index fingers rested on the two buttons of a joystick placed on a table in front of them. Pressing these buttons corresponded respectively to a "yes" (target present) or a "no" (target absent) answer. Participants were asked to respond whether a target was present or not during a stimulus presentation. They did not have to locate the target. Response times were measured from the onset of the stimulus presentation. Participants were instructed to respond as accurately as possible, but also as fast as possible. After each trial, feedback on performance was given, either by a high-pitched tone indicating a right answer, or by a lowpitched tone signaling a wrong answer. After each series of 64 trials, the experimenter started the next session. In between sessions, the participants were allowed a two minute break. When a block (representing a single condition) was completed, the participants took another brief moment to pause before the next block (condition) started. After finishing two series, the participants took a break which lasted minimally one hour before completing the final two series. The whole experiment lasted about two hours.

\section{RESULTS}

Mean response times, error rates and percentages of misses and false alarms were computed for each participant and each condition. In 11 (out of 4680 trials) cases the response time exceeded $2000 \mathrm{~ms}(0.2 \%)$. These data were removed from further analysis.

Fig. 3 shows the group's mean response times as a function of set size (number of activated tactors) for each of the 4 conditions (low versus high targe-nontarget similarity and targetnontarget roles interchanged). A repeated measures ANOVA of the individual mean response times showed a main effect of set size for target-present trials in all conditions, $(\mathrm{F}(3,15)=18.28, \mathrm{p}<.0001)$. Neither a main effect of condition, nor an interaction effect was found. 

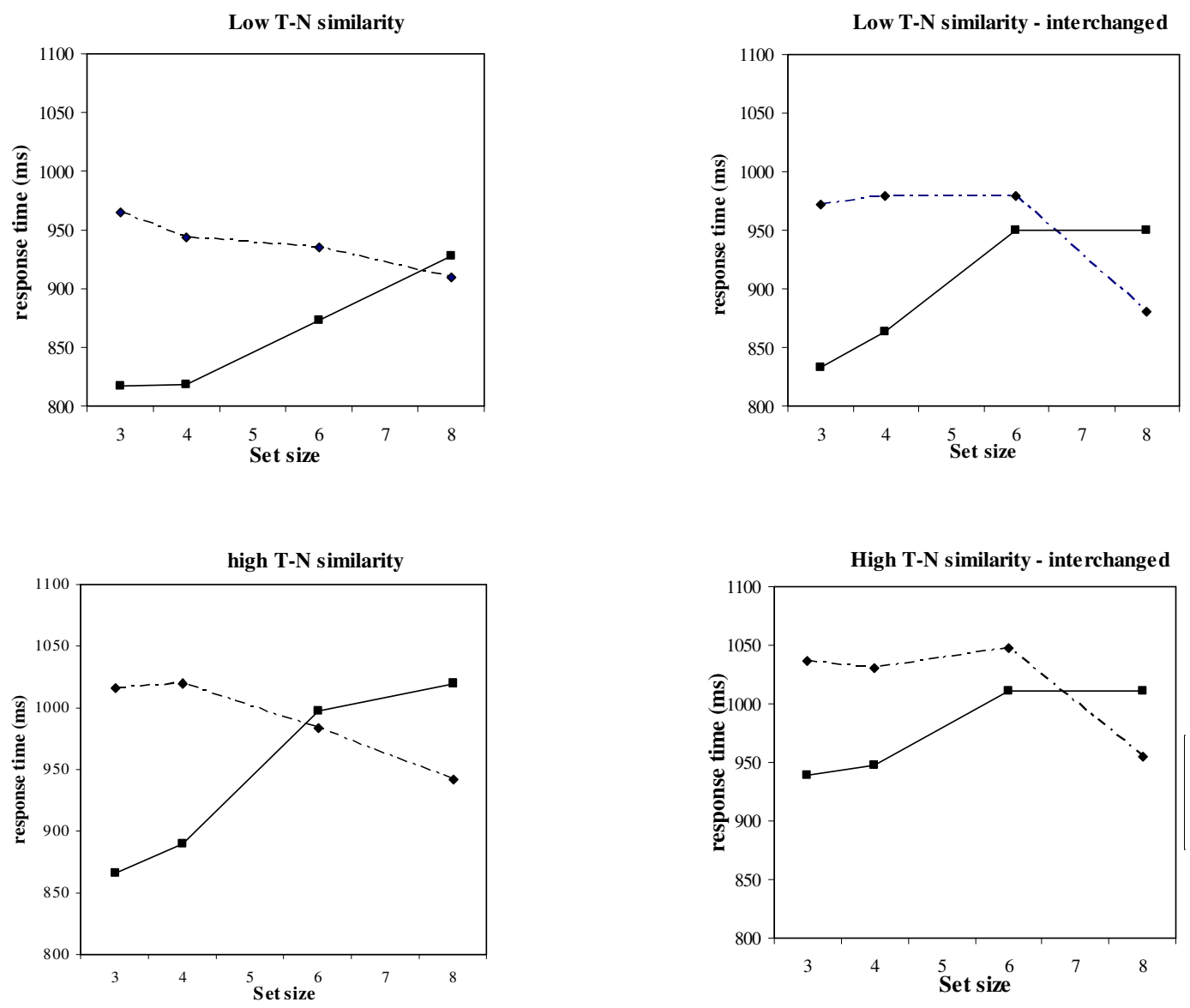

Fig. 3. Mean response times per condition for target present (solid lines) and target absent trials (dotted lines).

For the target-present trials, the mean response time increases with the number of nontargets. Using Helmert contrasts, significant differences were found between set size 3 and $6(\mathrm{~F}(1,5)=17.86, \mathrm{p}<.01)$, set size 3 and $8(\mathrm{~F}(1,5)=23.04, \mathrm{p}<$ $.005)$, set size 4 and $6(F(1,5)=18.28, p<.01)$, set size 4 and $8(F(1,5)=19.66, p<.01)$ and set size 6 and $8(F(1,5)=$ $7.85, \mathrm{p}<.05)$.

For the target-absent trials, the mean response time decreases with the number of nontargets $(\mathrm{F}(3,15)=5.60, \mathrm{p}<.01)$. However, this effect is mainly due to set size 8 , which causes a larger reduction in response time compared to the other set sizes, especially in the low T-N similarity - interchanged and the high T-N similarity - interchanged conditions (Fig. $6)$. When set size 8 is omitted from the analysis, no significant effect remains.

Fig. 4 shows the percentage of correct responses as a function of set size for target-present and target-absent trials. Notice that set size 8 only has a correct response percentage of only $65.6 \%$ in the high T-N similarity interchanged condition. 


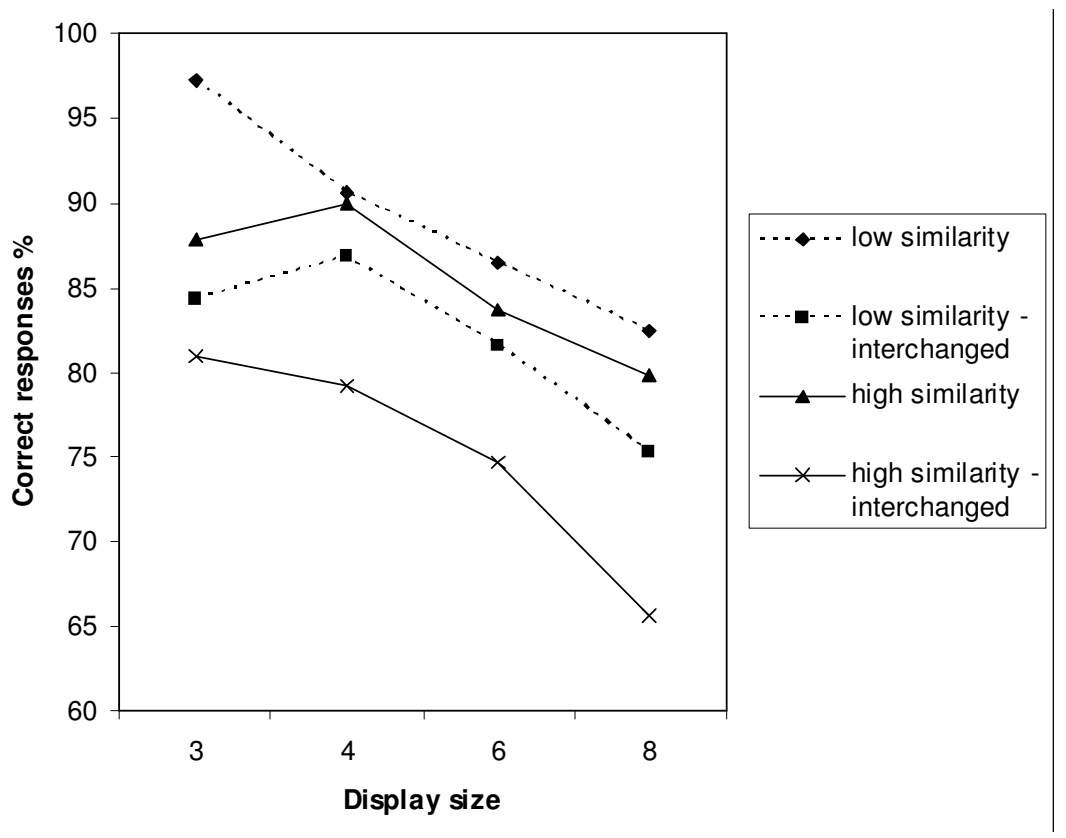

Fig. 4. Percentage correct responses as a function of set size.

From a further analysis of the percentages of misses and false alarms (by calculating d-prime and beta) we find a neutral response criterion (beta close to 1.0) for a small set size, and a conservative response criterion (beta close to 4: the participant is more likely to respond "target absent" most of the time) for large set sizes.

In perceptual-motor tasks, a tradeoff is usually observed between the speed with which a task is performed and the number of mistakes that are made. A participant can either perform the task very fast and make a large number of errors, or very slow and make few errors. When asked to perform a task as well as possible, people will deploy various strategies to optimize speed, accuracy, or a combination of the two. In this study, participants were asked to optimize their accuracy. To investigate the existence of a speed-accuracy trade-off (SAT), we made a SAT plot for every participant and analyzed the results by an ANOVA. We found no evidence for a speed accuracy trade-off.

\section{DISCUSSION}

We find different results in slope for target-present and target-absent trials. For target-present trials the response time increased with set size, suggesting a serial discrimination process. The increase in response time per item ranged between 14.5 to $30.8 \mathrm{~ms} /$ item. This temporal increment is similar to the 20 to $30 \mathrm{~ms} / \mathrm{item}$ increase in response time typically found in visual conjunction search tasks ${ }^{18}$. In contrast, the results of target-absent trials did not show significant differences in response time with variations in set size (when omitting set size 8 from the analysis), suggesting a parallel discrimination process. This finding agrees with another recent haptic search study ${ }^{27}$, but contrasts with similar studies investigating the visual target detection capability ${ }^{20,29}$.

Furthermore, we found no significant difference between high versus low T-N similarity. Likewise, no effects were found for T-N interchanged identities, so no evidence was obtained for a search asymmetry. Maybe the target and nontarget patterns used in this study were too similar to induce differences in $\mathrm{T}-\mathrm{N}$ interchanged identities and/or to induce a search asymmetry. Therefore, more variations of simultaneously presented stimuli have to be considered. Besides, with the hardware used in this study, stimuli could be varied in rhythm but not in intensity or vibration frequency. It may be useful to vary other parameters to test for differences in T-N similarity and in T-N interchanged identities. 
Set size of 8 was experienced as extremely difficult by the subjects, which is confirmed by the percentage correct responses (Fig. 4). The poor performance in this condition was primarily due to a high rate of misses, rather than false alarms, which suggests that subjects had a tendency to respond "target absent" in this configuration (on average beta was close to 4 in this condition). A possible explanation for the difficulty with set size 8 may be vibrotactile spatial summation $^{30}$. This results when the neural activity produced by one vibrotactile stimulus is increased by simultaneously presenting a second vibrotactile stimulus on an adjacent skin area ${ }^{31}$. In the present study, the target may have been strong enough to drown out the non-targets up to set size 6, but with set size 8 neural activity may have increased to such a high level that it overruled the target detection. A solution for spatial summation could be to spread out tactors over a broader skin area since physical separation is important for perceiving stimuli separately ${ }^{32}$. Another explanation for the change in detection behaviour might be that the non-targets made up a so-called Gestalt figure, which is specifically possible for set size 8 , since it is the only set size in which the first and the last tactor of the series had the same distance as the intermediate tactors, thus forming a closed circle around the abdomen. For set sizes 3, 4 and 6 on the other hand, there was a 'gap' in between the last and the first tactor, which may have prevented a Gestalt figure of non-targets.

Multiple synchronized stimulus onsets are likely to relate to a common event. For the visual system it is known that multiple synchronized abrupt onsets are perceived as a whole (the Gestalt principle of 'common fate'), even when they contain a target that contrasts strongly with all others on several non-temporal features ${ }^{33}$. The transient neural activity induced by the onset of a stimulus probably increases with the number of stimulus items. This may explain our current findings that it takes longer to segregate the target from a larger number of nontargets, and that the mean response times for target-present and target-absent trials become more similar for larger set sizes. The constant response time for target absent trials may reflect a ceiling effect, that may occur because observers need a fixed time to decide whether the target is indeed absent from the overall perceived tactile event (the tactile Gestalt).

\section{ACKNOWLEDGMENT}

The authors are indebted to Antoon Wennemers for writing the software for the experiment.

This research has been supported by the GATE project, funded by the Netherlands Organization for Scientific Research (NWO) and the Netherlands ICT Research and Innovation Authority (ICT Regie).

Effort sponsored by the Air Force Office of Scientific Research, Air Force Material Command, USAF, under grant number FA8655-06-1-3005. The U.S Government is authorized to reproduce and distribute reprints for Governmental purpose notwithstanding any copyright notation thereon. The views and conclusions contained herein are those of the authors and should not be interpreted as necessarily representing the official policies or endorsements, either expressed or implied, of the Air Force Office of Scientific Research or the U.S. Government.

\section{REFERENCES}

1. MacLean, K. and Enriquez, M., Perceptual design of haptic icons, In: I. Oakley, S. O'Modhrain \& F. Newell (Ed.), Proceedings of EuroHaptics 2003, pp. 27-1-27-13,2003.

2. van Erp, J.B.F. (2007). Tactile displays for navigation and orientation: perception and behaviour. Utrecht, The Netherlands: Utrecht University.

3. van Erp, J.B.F., van Veen, H.A.H.C., Jansen, C. and Dobbins, T., Waypoint navigation with a vibrotactile waist belt, ACM Transactions on Applied Perception, 2(2) ,pp. 106-117, 2005.

4. van Erp, J.B.F. and van Veen, H.A.H.C., Vibrotactile in-vehicle navigation system, Transportation Research Part F: Traffic Psychology and Behaviour, 7(4-5) ,pp. 247-256, 2005.

5. van Erp, J.B.F., Presenting directions with a vibro-tactile torso display, Ergonomics, 48(3) ,pp. 302-313, 2005.

6. van Erp, J.B.F. and van Veen, H.A.H.C., A multi-purpose tactile vest for astronauts in the international space station, In: I. Oakley, S. O'Modhrain \& F. Newell (Ed.), Proceedings of EuroHaptics 2003, pp. 54-1-54-4,2003.

7. van Veen, H.A.H.C. and van Erp, J.B.F., Tactile information presentation in the cockpit, In: S.A. Brewster \& R. Murray-Smith (Ed.), Haptic Human-Computer Interaction. Lecture Notes in Computer Science, pp. 174-181, Springer Verlag, Berlin, Heidelberg, Germany, 2001. 
8. Moorhead, I.R., Holmes, S. \& Furnell, A. Understanding multisensory integration for pilot spatial orientation, (Report QINETIQ/KI/CHS/TR042277), QinetiQ Ltd, Farnborough, UK, (2004).

9. Kooi, F.L., Toet, A., Tripathy, S.P. and Levi, D.M., The effect of similarity and duration on spatial interaction in peripheral vision, Spatial Vision, 8(2) ,pp. 255-279, 1994.

10. Toet, A. and Levi, D.M., The two-dimensional shape of spatial interaction zones in the parafovea, Vision Research, 32(7) ,pp. 1349-1357, 1992.

11. Beck, D.M. and Kastner, S., Stimulus context modulates competition in human extrastriate cortex, Nature Neuroscience, 8(8),pp. 1110-1116, 2005.

12. Yantis, S., How visual salience wins the battle for awareness, Nature Neuroscience, 8(8) ,pp. 975-977, 2005.

13. Meinecke, C. and Donk, M., Detection performance in pop-out tasks: nonmonotonic changes with display size and eccentricity, Perception, 31(5) ,pp. 591-602, 2002.

14. Treisman, A. and Gelade, G., A feature integration theory of attention, Cognitive Psychology, 12,pp. 97-136, 1980.

15. Nothdurft, H.C., Salience and target selection in visual search, Visual Cognition, 14(4-8), pp. 514-542, 2006.

16. Treisman, A. and Gormican, S., Feature analysis in early vision: evidence from search asymmetries, Psychological Review, 95 ,pp. 15-48, 1988.

17. Gordon, I.E., Interactions between items in visual search, Journal of Experimental Psychology, 76,pp. 348-355, 1968.

18. Duncan, J. and Humphreys, G.W., Visual search and stimulus similarity, Psychological Review, 96(3) ,pp. 433-458, 1989.

19. Rauschenberger, R. and Yantis, S., Perceptual encoding efficiency in visual search, Journal of Experimental Psychology: General, 135(1),pp. 116-131, 2006.

20. Treisman, A. and Souther, J., Search asymmetry: a diagnostic for preattentive processing of separable features, Journal of Experimental Psychology, 114 ,pp. 285-310, 1985.

21. Gescheider, G.A., Bolanowski, S.J., Jr. and Verrillo, R.T., Vibrotactile masking: effects of stimulus onset asynchrony and stimulus frequency, Journal of the Acoustical Society of America, 85(5), pp. 2059-2064, 1989.

22. Gescheider, G.A. and Verrillo, R.T., Contralateral enhancement and suppression of vibrotactile sensation, Perception \& Psychophysics, 32(1),pp. 69-74, 1982.

23. Loomis, J.M. and Apkarian-Stielau, P., A lateral masking effect in tactile and blurred visual letter recognition, Perception \& Psychophysics, 20 ,pp. 221-226, 1976.

24. Loomis, J.M., Counterexample to the hypothesis of functional similarity between tactile and visual pattern perception, Perception \& Psychophysics, 54(2), pp. 179-184, 1993.

25. Cholewiak, R.W. and Collins, A.A., Vibrotactile pattern discrimination and communality at several body sites, Perception \& Psychophysics, 57(5),pp. 724-737, 1995.

26. Loomis, A.H., Analysis of tactile and visual confusion matrices, Perception \& Psychophysics, 31(1), pp. 41-52, 1982.

27. Overvliet, K.E., Smeets, J.B.J. and Brenner, E., Parallel and serial search in haptics, Perception \& Psychophysics, 69(7) ,pp. 1059-1069, 2007.

28. Wagenaar, W.A., Note on the construction of digram-balanced Latin squares, Psychological Bulletin, 72(6) ,pp. 384-386, 1969.

29. Theeuwes, J., Perceptual selectivity is task dependent: evidence from selective search, Acta Psychologica, 74 ,pp. 81-99, 1990.

30. Craig, J.C., Vibrotactile spatial summation, Perception \& Psychophysics, 4(6), pp. 351-354, 1968.

31. von Békésy, G., Funneling in the nervous system and its role in loudness and sensation intensity on the skin, Journal of the Acoustical Society of America, 30 ,pp. 399-412, 1958.

32. Cholewiak, R.W., The perception of tactile distance: influences of body site, space, and time, Perception, 28(7) ,pp. 851-875, 1999.

33. Hancock, P.J.B. and Phillips, W.A., Pop-out from abrupt visual onsets, Vision Research, 44(19) ,pp. 2285-2299, 2004. 